\title{
Probing the origin of the microwave anomalous foreground (Corrigendum)
}

\author{
N. Ysard, M.-A. Miville-Deschênes, and L. Verstraete \\ Institut d'Astrophysique Spatiale (IAS), Université Paris-Sud, 91405 Orsay, France \\ e-mail: nathalie.ysard@ias.u-psud.fr
}

A\&A 509, L1 (2010), DOI: 10.1051/0004-6361/200912715

Key words. dust, extinction - ISM: general - errata, addenda

The Letter "Probing the origin of the microwave anomalous foreground" was published in A\&A 509, L1 (2010). This corrigendum corrects the legend of Fig. 5, where the linestyles (dotted and dashed) were inverted (Fig. 1). Consequently, the results presented in Sect. 7.4 and Fig. 17 in Hoang et al. (2011) are in good agreement with the results of Ysard et al. (2010), regarding both the peak frequencies of the spectra and their relative intensities.

\section{References}

Hoang, T., Lazarian, A., \& Draine, B. T. 2011, ApJ, 741, 87

Miville-Deschênes, M., Ysard, N., Lavabre, A., et al. 2008, A\&A, 490, 1093

Ysard, N., \& Verstraete, L. 2010, A\&A, 509, A12

Ysard, N., Miville-Deschênes, M. A., \& Verstraete, L. 2010, A\&A, 509, L1

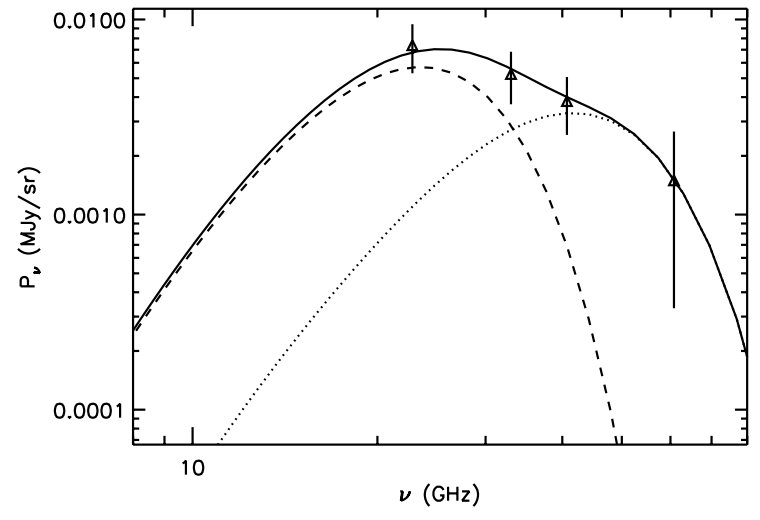

Fig. 1. A representative anomalous spectrum of our selected fields (taken from Model 4 of Miville-Deschênes et al. 2008). The solid line is the fit with our model (Ysard \& Verstraete 2010) comprising contributions from the CNM (10\%, dotted line) and from the WNM (90\%, dashed line). PAH parameters, see text of Ysard et al. (2010), are $m=$ $0.3 \mathrm{D}$, and $N_{\min }=24$ and 48 (CNM and WNM, respectively). 